\title{
Effect of Drop Jump Technique on the Reactive Strength Index
}

\author{
by \\ Artur Struzik ${ }^{1}$, Grzegorz Juras², Bogdan Pietraszewski ${ }^{3}$ Andrzej Rokita ${ }^{1}$
}

The basic drill of plyometric training aimed at improving lower limb power and jump height is a drop jump. This exercise can be performed using different techniques, which substantially affects jump variables. Therefore, the aim of this study was to compare the values of the reactive strength index (RSI) for countermovement drop jumps (CDJs) and bounce drop jumps (BDJs). The study was carried out in a group of 8 male youth basketball players. The tests were conducted using the AMTI BP600900 force plate to measure ground reaction forces and the Noraxon MyoMotion system to record kinematic data. Each player performed two CDJs and two BDJs from the height of 15, 30, 45 and 60 $\mathrm{cm}$. The RSI was calculated as a ratio of jump height and contact time. Moreover, the RSI was determined for the amortization and take-off phases separately. Significant differences $(p<0.05)$ between RSI values for CDJs and BDJs were recorded for jumps from 30, 45 and $60 \mathrm{~cm}$. Differences in RSI values for jumps from $15 \mathrm{~cm}$ were not significant. Furthermore, $C D J$ height values were significantly higher $(p<0.05)$ than the values recorded for BDJs. Times of contact, amortization and take-off during BDJs were significantly shorter $(p<0.05)$ than the respective values obtained for CDJs. Therefore, the use of the RSI to monitor plyometric training should be based on the drop jump technique that is commonly performed by basketball players.

Key words: countermovement drop jump, bounce drop jump, contact time, plyometrics, RSI.

\section{Introduction}

Basketball is a sport dominated by "explosive" movements such as rapid cuts and stops, sudden accelerations, change of directions or jumps. Therefore, effective performance requires an adequate level of lower limb power (Alemdaroğlu, 2012; Litkowycz et al., 2010) of which improvement is possible through the application of plyometric training based mainly on jumping exercises (Bobbert, 1990; Litkowycz et al., 2010; Pietraszewski and Struzik, 2013; Young et al., 1999).

The basic drill of plyometric training aimed at improving lower limb power and jump height is a drop jump (DJ) (Bobbert, 1990; Markovic, 2007; Markovic and Mikulic, 2010). This exercise can be performed using different techniques, which substantially affects jump variables. A bounce drop jump (BDJ) is aimed to reverse downward velocity into an upward one as soon as possible after landing. On the contrary, a countermovement drop jump (CDJ), of which aim is to achieve the highest possible jump height, requires making a larger downward movement upon landing. Therefore, the CDJ is similar to the countermovement jump (CMJ) (Bobbert et al., 1987; Bobbert, 1990; Young et al., 1995; Young et al., 1999). Obviously, it is possible to combine the $\mathrm{CDJ}$ and BDJ techniques to perform a jump with the maximum height and minimum ground contact time - DJ-H/t (Hunter and Marshall, 2002; Markwick et al., 2015; Young et al., 1995; Young et al., 1999).

\footnotetext{
1 - Department of Team Sport Games, University School of Physical Education, Wrocław, Poland.

2 - Department of Motor Behavior, Academy of Physical Education, Katowice, Poland.

3 - Department of Biomechanics, University School of Physical Education, Wrockaw, Poland.
} 
Different DJ technique may cause different training adaptations. The BDJ mainly improves lower limb power, whereas the CDJ is oriented to improvement in jump height and coordination (Bobbert et al., 1987; Bobbert, 1990; Young et al., 1995; Young et al., 1999). Young et al. (1999) documented significantly higher values of reactive strength after a 6 week training program based on DJ-H/t jumps. No such effect was observed in the group that followed a protocol based on the CDJ. Furthermore, Markovic (2007) suggested that the effect of plyometric training was likely to have higher impact on vertical jump height in "slow" stretch-shortening cycle (SSC) jumps (like the CMJ; the CDJ can be also added to this group) rather than in either concentric (squat jump - SJ) or "fast” SSC jumps (like BDJ). Marshall and Moran (2013) found that eight weeks of CDJ training enhanced significantly CMJ height, while a similar regime of the BDJ training did not.

Bobbert et al. (1987) showed that the mechanical output at knee and ankle joints reached larger values during the $\mathrm{BDJ}$ than $\mathrm{CDJ}$. Arampatzis et al. (2001) found that leg and ankle stiffness values were higher when the contact time during the DJ (directed by verbal instructions) was shorter. Furthermore, Cressey et al. (2007) demonstrated that lower-body unstable surface training lead to a higher increase in power output for the BDJ compared to CDJ. Differences in technique and variables that describe DJs also occur for different heights of the platform from which the drop is performed (Ball et al., 2010) or depending on the gender of the subjects (Laffaye and Choukou, 2010). Bobbert (1990) concluded that $\mathrm{DJ}$ technique was the most important variable to be controlled to ensure adequate training effects. Furthermore, Walsh et al. (2004) also found that manipulation of jump technique played a greater role in control of important jump variables than the platform height.

Since jumping technique has a substantial effect on jumping variables, it is necessary to employ various standards for indices that control variables of the DJ. An example of the index used for the DJ which is susceptible to jumping technique is a reactive strength index (RSI). The RSI is the quotient of the jump height and contact time (McClymont and Hore, 2003). Theoretically, a higher value of the RSI reflects a more efficient performance of the movement in the stretch- shortening cycle. The value of the RSI can also change (increase) following plyometric training (Lloyd et al., 2012).

Therefore, the aim of this study was to compare the values of the RSI for CDJs and BDJs. If the differences between the values of the RSI turned out to be substantial, this may suggest that DJ variables should be carefully controlled (including DJ technique) in a training cycle. According to the aforementioned reports, CDJs should be characterized by greater height of the jump and longer time of the contact phase compared to BDJs. Therefore, maximal values of flexion in the lower limb and upper body joints should be significantly lower than for CDJs. Finding significant differences between CDJs and BDJs for the variables of the jump height, contact time and the values of flexion in lower limb and upper body joints leads to the conclusion that the subjects were able to perform the above types of jumps using the demanded movement technique.

\section{Material and Methods}

\section{Participants}

The study was carried out in a group of 8 male youth basketball players (age: $17.0 \pm 0.2$ years; body height: $188.4 \pm 6.4 \mathrm{~cm}$; body mass: 79.6 $\pm 7.4 \mathrm{~kg}$; training experience: $4.4 \pm 1.6$ years). The research project was approved by the Ethics Committee of the University School of Physical Education in Wrocław, Poland, and the procedures complied with the Declaration of Helsinki regarding human experimentation.

\section{Measures}

The tests were conducted using an AMTI BP600900 force plate (Watertown, MA, USA) to measure ground reaction forces and the Noraxon MyoMotion system (Scottsdale, AZ, USA) to record kinematic data: cervical flexion-extension, lumbar flexion-extension, thoracic flexionextension, hip flexion-extension, knee flexionextension and ankle dorsi-plantar flexion angles.

\section{Procedures}

Before the tests, each participant was familiarized with the research goals. A $10 \mathrm{~min}$ warm-up was administered prior to the measurements. Each player performed jumping exercises in the following order: two CDJs and two BDJs from the height of 15, 30, 45 and $60 \mathrm{~cm}$ (in sequence), with hands on the hips and elbows bowed outward (total 16 jumps with a break after 
8 jumps and a re-warm-up). In the case of the CDJ, the participants were instructed to: „jump as high as possible after the drop off from the platform". Furthermore, for the BDJ, the athletes were instructed to: "jump up as fast as possible after contact with the ground and make sure that the jump is the highest possible". To initiate the drop action, participants were asked to "step out" from the platform one foot at a time (right lower limb first). This instruction prevented from jumping out from the platform. The participant was instructed to perform the contact phase (amortization + take-off) and landing phase on the force plate. The better results achieved for each jump were registered for further analysis.

MyoMotion Reseach inertial sensors were placed according to the rigid-body model with 16 joint segments used in MR3 software on the right shoe (top of the upper foot, slightly below the ankle), right shank (frontal on the tibia bone), right thigh (frontal attachment on a lower quadrant of quadriceps, slightly above the knee cap, area of lowest muscle belly displacement in motion), pelvic (bony area of sacrum), lower thoracic (on the spinal cord at approximately L1/T12, the strap belt on the front body side positioned on lower ribs), upper thoracic (below C7 along the spinal cord, but high enough not to be affected by upper trapezius muscle movement) and head (in the middle of the back of the head). Calibration was carried out in an upright position of the subject in order to determine the value of the $0^{\circ}$ angle in the joints studied. Sampling frequency for the inertial sensors was set at 200 $\mathrm{Hz}$. Instantaneous changes in joint angles were recorded with respect to the transverse axis during jumps.

Recording of ground reaction forces during jumps allowed for determination of the time of contact and flight $\left(t_{f}\right)$ phases. Consequently, the jump height $(h)$ was calculated using the following formula:

$$
h=\frac{1}{8} g \cdot t_{f}^{2},
$$

where $g$ is the acceleration due to gravity. The RSI was calculated as a ratio of jump height $(\mathrm{mm})$ and contact time (ms) (McClymont and Hore, 2003):

$$
R S I=\frac{\text { jump height }}{\text { contact time }} .
$$

Recording the instantaneous changes in the knee joint angle during performance of jumps allowed for division of time of contact with the surface into amortization time $\left(t_{a}\right)$ and take-off time $\left(t_{t}\right)$. Thus, the RSI could also be calculated for the amortization and take-off phases separately (Pietraszewski and Rutkowska-Kucharska, 2011; Struzik et al., 2016):

$$
\begin{gathered}
R S I(\text { amortization })=\frac{h}{t_{a}}, \\
R S I(\text { push }- \text { off })=\frac{h}{t_{t}} .
\end{gathered}
$$

The sampling rate for the signal from the force plate was set at $100 \mathrm{~Hz}$. Compared to previous reports, RSI calculations and drop jump height measurements had acceptable reliability (Arteaga et al., 2000; Beattie and Flanagan, 2015; Markwick et al., 2015).

\section{Statistical Analysis}

Normality of distribution of each variable was tested using the Shapiro-Wilk and Lilliefors tests. Since the data was not normally distributed, the Wilcoxon matched-pairs test was applied in order to evaluate the differences between the variables describing CDJs and BDJs. Statistical significance was determined at an alpha level of 0.05 .

\section{Results}

Table 1 presents mean values $( \pm$ SD) of jumping variables and the RSI during CDJs and BDJs. Statistically significant differences $(p<0.05)$ between RSI values for CDJs and BDJs were recorded for jumps from 30, 45 and $60 \mathrm{~cm}$. Differences in RSI values for jumps from $15 \mathrm{~cm}$ were not statistically significant. Furthermore, CDJ height values were significantly higher $(p<$ 0.05 ) than the values recorded for BDJs. Time of contact, amortization and take-off during BDJs was significantly shorter $(p<0.05)$ than the respective values obtained for CDJs.

Table 2 shows mean values $( \pm$ SD) of maximum angles during CDJs and BDJs. Positive values of the angles correspond to flexion and dorsi-flexion. Statistically significant differences $(p$ $<0.05$ ) were found between CDJs and BDJs in maximum values of angles of flexion in the knee and hip joints (from the height of 15, 30, 45 and 60 $\mathrm{cm}$ ) and thoracic extension (from the height of 15, 30 and $45 \mathrm{~cm}$ ). The angles of flexion in the knee and hip joints were greater during CDJs. The absolute values of thoracic extension angles for CDJs were also greater. Differences for the cervical, lumbar and ankle joints were not significant. 
Table 1

Mean values ( $\pm S D$ ) of jumping variables and RSI for $C D J$ s and BDJs.

\begin{tabular}{|c|c|c|c|c|}
\hline $\begin{array}{l}\text { Platform height } \\
(\mathrm{cm})\end{array}$ & Jumping variables & CDJ & BDJ & $\Delta$ \\
\hline \multirow{7}{*}{15} & RSI & $1.04 \pm 0.2$ & $1.17 \pm 0.21$ & $0.13 \pm 0.29$ \\
\hline & RSI (amortization) & $2.24 \pm 0.64$ & $2.65 \pm 0.54$ & $0.41 \pm 0.76$ \\
\hline & RSI (take-off) & $1.95 \pm 0.29$ & $2.11 \pm 0.36$ & $0.15 \pm 0.47$ \\
\hline & contact time (s) & $0.33 \pm 0.08$ & $0.24 \pm 0.02$ & $0.09 \pm 0.09^{*}$ \\
\hline & amortization time (s) & $0.16 \pm 0.05$ & $0.11 \pm 0.01$ & $0.05 \pm 0.05^{*}$ \\
\hline & take-off time (s) & $0.17 \pm 0.04$ & $0.13 \pm 0.01$ & $0.04 \pm 0.04^{*}$ \\
\hline & jump height (m) & $0.33 \pm 0.05$ & $0.28 \pm 0.04$ & $0.06 \pm 0.03^{*}$ \\
\hline \multirow{7}{*}{30} & RSI & $0.91 \pm 0.11$ & $1.24 \pm 0.24$ & $0.33 \pm 0.20^{*}$ \\
\hline & RSI (amortization) & $1.95 \pm 0.27$ & $2.75 \pm 0.63$ & $0.79 \pm 0.52^{*}$ \\
\hline & RSI (take-off) & $1.72 \pm 0.24$ & $2.27 \pm 0.43$ & $0.55 \pm 0.34^{*}$ \\
\hline & contact time (s) & $0.39 \pm 0.06$ & $0.23 \pm 0.03$ & $0.16 \pm 0.07^{*}$ \\
\hline & amortization time (s) & $0.18 \pm 0.03$ & $0.10 \pm 0.02$ & $0.08 \pm 0.03^{*}$ \\
\hline & take-off time (s) & $0.21 \pm 0.04$ & $0.12 \pm 0.02$ & $0.08 \pm 0.04^{*}$ \\
\hline & jump height (m) & $0.35 \pm 0.04$ & $0.28 \pm 0.03$ & $0.08 \pm 0.04^{*}$ \\
\hline \multirow{7}{*}{45} & RSI & $0.91 \pm 0.21$ & $1.19 \pm 0.31$ & $0.28 \pm 0.31^{*}$ \\
\hline & RSI (amortization) & $1.97 \pm 0.47$ & $2.57 \pm 0.71$ & $0.60 \pm 0.70^{*}$ \\
\hline & RSI (take-off) & $1.72 \pm 0.42$ & $2.22 \pm 0.55$ & $0.50 \pm 0.56^{*}$ \\
\hline & contact time (s) & $0.40 \pm 0.09$ & $0.25 \pm 0.05$ & $0.15 \pm 0.09^{*}$ \\
\hline & amortization time (s) & $0.19 \pm 0.04$ & $0.12 \pm 0.03$ & $0.07 \pm 0.05^{*}$ \\
\hline & take-off time (s) & $0.21 \pm 0.05$ & $0.13 \pm 0.03$ & $0.08 \pm 0.05^{*}$ \\
\hline & jump height (m) & $0.35 \pm 0.05$ & $0.28 \pm 0.04$ & $0.07 \pm 0.02^{*}$ \\
\hline \multirow{7}{*}{60} & RSI & $0.85 \pm 0.19$ & $1.11 \pm 0.2$ & $0.25 \pm 0.21^{*}$ \\
\hline & RSI (amortization) & $1.83 \pm 0.39$ & $2.42 \pm 0.47$ & $0.59 \pm 0.46^{*}$ \\
\hline & RSI (take-off) & $1.61 \pm 0.38$ & $2.04 \pm 0.35$ & $0.43 \pm 0.40^{*}$ \\
\hline & contact time (s) & $0.43 \pm 0.08$ & $0.28 \pm 0.04$ & $0.15 \pm 0.09^{*}$ \\
\hline & amortization time (s) & $0.20 \pm 0.04$ & $0.13 \pm 0.02$ & $0.07 \pm 0.04^{*}$ \\
\hline & take-off time (s) & $0.23 \pm 0.05$ & $0.15 \pm 0.02$ & $0.08 \pm 0.05^{*}$ \\
\hline & jump height (m) & $0.35 \pm 0.04$ & $0.30 \pm 0.04$ & $0.05 \pm 0.02^{*}$ \\
\hline
\end{tabular}

$C D J$ - countermovement drop jump, BDJ-bounce drop jump,

$R S I$ - reactive strength index,

$\Delta$-difference between variables that describe CDJs and BDJs,

* - significant differences at $p<0.05$ 
Table 2

Mean values ( $\pm S D$ ) of maximum angles during $C D J s$ and $B D J s$.

\begin{tabular}{|c|c|c|c|c|}
\hline $\begin{array}{l}\text { Platform height } \\
(\mathrm{cm})\end{array}$ & Joint movement & $\mathrm{CDJ}\left({ }^{\circ}\right)$ & $\mathrm{BDJ}\left({ }^{\circ}\right)$ & $\Delta\left({ }^{\circ}\right)$ \\
\hline \multirow{6}{*}{15} & cervical flexion-extension & $-27.1 \pm 4.9$ & $-25.4 \pm 9.4$ & $1.7 \pm 11.4$ \\
\hline & lumbar flexion-extension & $35.3 \pm 8.0$ & $29.5 \pm 10.7$ & $5.8 \pm 7.1$ \\
\hline & thoracic flexion-extension & $-10.4 \pm 12.2$ & $2.1 \pm 12.9$ & $12.5 \pm 14.3^{*}$ \\
\hline & hip flexion-extension & $44.2 \pm 14.2$ & $30.3 \pm 11.0$ & $13.9 \pm 10.5^{*}$ \\
\hline & knee flexion-extension & $74.9 \pm 9.5$ & $58.1 \pm 16.3$ & $16.9 \pm 13.6^{*}$ \\
\hline & ankle dorsi-plantar flexion & $24.5 \pm 6.7$ & $18.5 \pm 12.5$ & $6.0 \pm 10.1$ \\
\hline \multirow{6}{*}{30} & cervical flexion-extension & $-36.5 \pm 4.5$ & $-26.4 \pm 19.8$ & $10.0 \pm 18.2$ \\
\hline & lumbar flexion-extension & $38.2 \pm 8.8$ & $29.5 \pm 11.5$ & $8.6 \pm 11.3$ \\
\hline & thoracic flexion-extension & $-12.4 \pm 0.24$ & $6.0 \pm 9.8$ & $18.4 \pm 11.9^{*}$ \\
\hline & hip flexion-extension & $58.2 \pm 13.2$ & $23.1 \pm 24.8$ & $29.7 \pm 23.5^{*}$ \\
\hline & knee flexion-extension & $85.3 \pm 18.3$ & $54.8 \pm 16.0$ & $30.5 \pm 17.3^{*}$ \\
\hline & ankle dorsi-plantar flexion & $21.7 \pm 5.9$ & $19.1 \pm 10.4$ & $2.6 \pm 9.3$ \\
\hline \multirow{6}{*}{45} & cervical flexion-extension & $-34.4 \pm 7.1$ & $-24.5 \pm 23.0$ & $9.9 \pm 19.1$ \\
\hline & lumbar flexion-extension & $39.4 \pm 8.8$ & $34.0 \pm 15.3$ & $5.4 \pm 12.5$ \\
\hline & thoracic flexion-extension & $-7.9 \pm 15.4$ & $3.1 \pm 11.8$ & $11.0 \pm 9.6^{*}$ \\
\hline & hip flexion-extension & $54.5 \pm 17.7$ & $21.0 \pm 27.5$ & $33.5 \pm 29.1^{*}$ \\
\hline & knee flexion-extension & $85.4 \pm 13.8$ & $57.7 \pm 20.4$ & $27.7 \pm 16.8^{*}$ \\
\hline & ankle dorsi-plantar flexion & $20.9 \pm 11.5$ & $16.8 \pm 10.4$ & $4.0 \pm 17.6$ \\
\hline \multirow{6}{*}{60} & cervical flexion-extension & $-34.7 \pm 9.2$ & $-27.7 \pm 15.4$ & $7.0 \pm 13.2$ \\
\hline & lumbar flexion-extension & $41.5 \pm 10.0$ & $33.7 \pm 12.1$ & $7.8 \pm 12.9$ \\
\hline & thoracic flexion-extension & $-9.1 \pm 15.5$ & $-3.8 \pm 9.5$ & $5.3 \pm 18.9$ \\
\hline & hip flexion-extension & $59.6 \pm 14.2$ & $31.6 \pm 13.9$ & $27.9 \pm 14.9^{*}$ \\
\hline & knee flexion-extension & $90.3 \pm 15.1$ & $61.6 \pm 20.3$ & $28.7 \pm 17.7^{*}$ \\
\hline & ankle dorsi-plantar flexion & $18.8 \pm 5.7$ & $12.0 \pm 15.0$ & $6.7 \pm 15.6$ \\
\hline
\end{tabular}

$C D J$ - countermovement drop jump, BDJ - bounce drop jump,

$\Delta$-difference between maximum angles during $C D J s$ and $B D J s$,

* - significant differences at $p<0.05$

\section{Discussion}

The technique of DJ performance causes that CDJs are higher than BDJs, with elongation of the contact time (Table 1). In the present study, the increased contact time resulted from the elongation of both the amortization and take-off phases. This is consistent with previous reports concerning influence of DJ performance instruction (Bobbert et al., 1987; Bobbert, 1990; 
Young et al., 1995; Young et al., 1999). Differences in time of contact with the ground are also accompanied by the differences in maximal angles of flexion in knee and hip joints and thoracic extension. Longer time of the take-off during CDJs leads to an increase in angles of flexion in the knee and hip joints and thoracic extension. The occurrence of these relationships has been suggested in studies of Bobbert et al. (1987), Bobbert (1990) and Markovic (2007). Greater height of CDJs compared to BDJs may result from a greater range of motion in the knee, hip and thoracic joints that allows to obtain greater angular velocities in these joints (Bober et al., 1987; Bobbert, 1990). However, differences in the techniques of CDJ and BDJ performance do not induce significant differences in maximum values of angles during movements in the cervical and lumbar joints and, surprisingly, the ankle joint. Based on the differences recorded for the variables that describe $\mathrm{CDJs}$ and $\mathrm{BDJs}$, it can be concluded that the study participants performed both types of jumps effectively. Therefore, it may be stated that the technique of the DJ is affected by the instruction to perform the exercise (Young et al., 1995; Jidovtseff et al., 2014; Khuu et al., 2015; Louder et al., 2015).

Some training studies have found that plyometric training based on DJs does not significantly enhance jump height or lower limb power, while other reports showed a large variability in the magnitude of such enhancement. Several factors, including a training program design (the type of exercises, training duration, training frequency, volume and intensity of training), subject characteristics (age, gender, fitness level, sport practice) and methods of testing different types of vertical jumps may be responsible for the conflicting findings concerning plyometric training. However, potentially inconsistent effects of DJ training may result from the differences in the DJ technique employed (Bobbert, 1990; Markovic, 2007; Markovic and Mikulic, 2010).

The RSI is mostly used to profile stretchload tolerance and to identify appropriate platform heights for the performance of drop jumps. The modification of the RSI (RSI take-off) allows not only for a more detailed control of the athlete over the training process, but also for its utilization for the CMJ (Pietraszewski and Struzik,
2013; Pietraszewski et al., 2015; Suchomel et al., 2015). Statistically significant differences in jump height, contact, amortization and take-off times between CDJs and BDJs induce significant differences, also in RSI value (for DJ from 30, 45 and $60 \mathrm{~cm}$ ). Differences in RSI values for jumps from $15 \mathrm{~cm}$ were not significant, which can be explained by a relatively low height of the platform. Bobbert et al. (1986) observed that when performing a DJ, some subjects spontaneously chose to make a large amplitude movement after a drop (CDJ), whereas others preferred a small amplitude movement (BDJ). If drop height is increased and jump technique is not controlled, subjects are likely to make a larger downward movement upon landing. Therefore, the DJ technique will shift from the BDJ to CDJ (Bobbert, 1990; Hunter and Marshall, 2002).

To conclude, the use of the RSI to monitor plyometric training should refer to the DJ expected performance technique. It is also necessary to control DJ technique since performing a jump which does not meet the specific criteria may impact jumping variables and RSI values. It should be expected that RSI values will be slightly higher for BDJs than CDJs. Due to differences in jumping technique, DJs from the same platform height can yield different jump variables. Depending on the needs, the choice of the jumping technique allows for improving various jumping variables.

The limitations of this study may be a small size and type (young basketball players) of the studied group. It should be also emphasized that the use of the force plate allowed computation of the jump height based on the duration of the flight phase, which eliminated the effect of individual variables (such as foot or upper limb length). We realize that determination of the jump height based on the flight phase duration has some limitations and is not a perfect method. However, the measurement error is relatively small compared to other currently used methods. 


\section{Acknowledgements}

The results from this study were presented in the abstract form at the conference: „International Conference of the Polish Society of Biomechanics, Biomechanics 2016, 5-7 september 2016" and published in the book of abstracts.

\section{References}

Alemdaroğlu U. The relationship between muscle strength, anaerobic performance, agility, sprint ability and vertical jump performance in professional basketball players. J Hum Kinet, 2012; 31: 149-158

Arampatzis A, Schade F, Walsh M, Bruggemann G-P. Influence of leg stiffness and its effect on myodynamic jumping performance. J Electromyogr Kines, 2001; 11(5): 355-364

Arteaga R, Dorado C, Chavarren J, Calbet JAL. Reliability of jumping performance in active men and woman under different stretch loading conditions. J Sport Med Phys Fit, 2000; 40(1): 26-34

Ball NB, Stock CG, Scurr JC. Bilateral contact ground reaction forces and contact times during plyometric drop jumping. J Strength Cond Res, 2010; 24(10): 2762-2769

Beattie K, Flanagan EP. Establishing the reliability \& meaningful change of the drop-jump reactive-strength index. J Aust Strength Cond, 2015; 23(5): 12-18

Bobbert MF. Drop jumping as a training method for jumping ability. Sports Med, 1990; 9(1): 7-22

Bobbert MF, Huijing PA, van Ingen Schenau GJ. Drop jumping. I. The influence of jumping technique on the biomechanics of jumping. Med Sci Sport Exer, 1987; 19(4): 332-338

Bobbert MF, Mackay M, Schinkelshoek D, Huijing A, van Ingen Schenau GJ. Biomechanical analysis of drop and countermovement jumps. Eur J Appl Physiol, 1986; 54(6): 566-573

Bober T, Putnam CA, Woodworth GG. Factors influencing the angular velocity of a human limb segment. $J$ Biomech, 1987; 20(5): 511-521

Cressey EM, West CA, Tiberio DP, Kraemer WJ, Maresh CM. The effects of ten weeks of lower-body unstable surface training on markers of athletic performance. J Strength Cond Res, 2007; 21(2): 561-567

Hunter JP, Marshall RN. Effects of power and flexibility training on vertical jump technique. Med Sci Sports Exer, 2002; 34(3): 478-486

Jidovtseff B, Quievre J, Harris NK, Cronin JB. Influence of jumping strategy on kinetic and kinematic variables. J Sport Med Phys Fit, 2014; 54(2): 129-138

Khuu S, Musalem LL, Beach TAC. Verbal instructions acutely affect drop vertical jump biomechanicsimplications for athletic performance and injury risk assessments. J Strength Cond Res, 2015; 29(10): 2816-2826

Laffaye G, Choukou MA. Gender bias in the effect of dropping height on jumping performance in volleyball players. J Strength Cond Res, 2010; 24(8): 2143-2148

Litkowycz R, Słomka K, Grygorowicz M, Król H. The influence of plyometrics training on the maximal power of the lower limbs in basketball players aged 16-18. Antropomotoryka, 2010; 49: 33-44

Lloyd RS, Oliver JL, Hughes MG, Williams CA. The effects of 4-weeks of plyometric training on reactive strength index and leg stiffness in male youths. J Strength Cond Res, 2012; 26(10): 2812-2819

Louder T, Bressel M, Bressel E. The kinetic specificity of plyometric training: verbal cues revisited. J Hum Kinet, 2015; 49: 201-208

Markovic G. Does plyometric training improve vertical jump height? A meta-analytical review. Br J Sports Med, 2007; 41(6): 349-355

Markovic G, Mikulic P. Neuro-musculoskeletal and performance adaptations to lower-extremity plyometric training. Sports Med, 2010; 40(10): 859-895

Markwick WJ, Bird SP, Tufano JJ, Seitz LB, Haff GG. The intraday reliability of the reactive strength index calculated from a drop jump in professional men's basketball. International Journal of Sports Physiology 
and Performance, 2015; 10(4): 482-488

Marshall BM, Moran KA. Which drop jump technique is most effective at enhancing countermovement jump ability, "countermovement" drop jump or „bounce" drop jump? J Sport Sci, 2013; 31(12): 1368-1374

McClymont D, Hore A. Use of the reactive strength index RSI as a plyometric monitoring tool. $5^{\text {th }}$ World Congress of Science in Football, Lisbon; 2003

Pietraszewski B, Siemieński A, Bober T, Struzik A, Rutkowska-Kucharska A, Nosal J, Rokita A. Lower extremity power in female soccer athletes: a pre-season and in-season comparison. Acta Bioeng Biomech, 2015; 17(3): 129-135

Pietraszewski B, Struzik A. Evaluation of selected biomechanical parameters in female team sports players. Acta Bioeng Biomech, 2013; 15(4): 103-108

Pietraszewski B, Rutkowska-Kucharska A. Relative power of lower limbs in drop jump. Acta Bioeng Biomech, 2011; 13(1): 13-18

Struzik A, Pietraszewski B, Bober T. Relationships between the H/Q ratio and variables describing CMJ and DJ jumps. Mitteilungen Klosterneuburg, 2016; 66(2): 123-133

Suchomel TJ, Sole CJ, Bailey CA, Grazer JL, Beckham GK. A comparison of reactive strength index-modified between six U.S. collegiate athletic teams. J Strength Cond Res, 2015; 29(5): 1310-1316

Walsh M, Arampatzis A, Schade F, Brüggemann G-P. The effect of drop jump starting height and contact time on power, work performed, and moment of force. J Strength Cond Res, 2004; 18(3): 561-566

Young WB, Pryor JF, Wilson GJ. Effect of instructions on characteristics of countermovement and drop jump performance. J Strength Cond Res, 1995; 9(4): 232-236

Young WB, Wilson GJ, Byrne C. A comparison of drop jump training methods: effect on leg extensor strength qualities and jumping performance. Int J Sports Med, 1999; 20(5): 295-303

\section{Corresponding author:}

\section{Artur Struzik,}

Department of Team Sport Games,

University School of Physical Education, 1. Mickiewicza 58, 51-684 Wrocław, Poland

Phone/fax.: +48 713473551 / 713473562

E-mail: artur.struzik@awf.wroc.pl 